\title{
Avifauna alojada nos CETAS/IBAMA nos estados do Rio Grande do Norte e Paraíba, Brasil
}

A comercialização ilegal de animais silvestres é um processo disseminado por todo o mundo. No Brasil, essa prática ilegal baseada na retirada dos animais silvestres da natureza se tornou uma das principais fontes do contrabando da fauna. Por representar um dos grupos de vertebrados de maior importância cinegética no mundo, sobretudo, devido a sua relevância econômica e sociocultural, as aves são os animais que mais sofrem com o tráfico clandestino. $O$ presente estudo fo realizado com objetivo de caracterizar o comércio ilegal de aves silvestres com base no registro de dados dos Centros de Triagem de Animais Silvestres do IBAMA em duas áreas do Nordeste do Brasil - Rio Grande do Norte e Paraíba. A coleta de dados ocorreu de março de 2014 a janeiro de 2015 através de consultas aos bancos informacionais disponibilizado pelo CETAS-RN e pelo CETAS-PB. Os dados foram quantificados entre os anos de 2005 a 2014 , referentes à entrada de aves apreendidas ou entregues voluntariamente pela população local. Pode-se estimar que houve o aumento do tráfico de animais silvestres devido: a redução no recebimento de espécies nos CETAS de ambos os Estados; a falta de investimentos e a carência de destinação desses animais. Houve prevalência das aves entre os grupos apreendidos e recebidos, destacando-se os Passeriformes e os Psittaciformes. As espécies mais apreendidas foram o Sicalis flaveola (canário-da-terra) Paroaria dominicana (galo-de-campina) e Cyanoloxia brissonii (azulão). Já as espécies endêmicas da caatinga mais frequentes durante os anos analisados foram P. dominicana (galo-de-campina), Eupsittula cactorium (periquito-da-caatinga) e Sporophila albogularis (golinha).

Palavras-chave: Aves; Comércio llegal; Fiscalização.

\section{Birdlife hosted in the CETAS/IBAMA in Rio Grande do Norte and Paraíba States, Brazil}

\begin{abstract}
The illegal commercialization of wild animals is a disseminated process all over the world. In Brazil this illegal practice, based on the removal of wild animals from nature, has become one of the main sources of the fauna's smuggling. For representing one of the vertebrate groups of great synergetic importance in the world, especially due to its economic and sociocultural relevance, the birds are the animals which suffer the most with the clandestine smuggling. This study was made aiming to characterize the illegal trade of wild birds based on the registered data from the CETAS (Wild Animal Sorting Centres) of IBAMA in two areas in the Brazil's Northeast - Rio Grande do Norte and Paraiba. The data collection took place from March 2014 to January 2015, through the database query provided by CETAS RN and CETAS-PB. The information was qualified between the 2005 and 2014, relating to the entrance of seized or voluntarily handed over birds by the local population. One can estimate that there was an increase of wild animal's smuggling due to: the reduction on the species receipt in the CETAS of both States; the lack of investments and absence of destination for those animals. There was prevalence of birds among seized and handed over groups, standing out the Passeriformes and Psittaciformes. The most seized species were the Sicalis flaveola (canary), Paroaria dominicana (red-cowled cardinal) and Cyanoloxia brissonii (ultramarine grosbeak). During the analysed years the more frequent endemic species of caatinga were: P. dominicana (red-cowled cardinal), Eupsittula cactorium (cactus parakeet) e Sporophila albogularis (white-throated seedeater).
\end{abstract}

Keywords: Birds; Illegal Trade; Monitoring.

Topic: Conservação da Biodiversidade

Reviewed anonymously in the process of blind peer.
Received: 11/09/2019

Approved: 20/11/2019
Thatiany de Sousa Pereira

Universidade Federal de Campina Grande, Brasil

http://lattes.cnpq.br/5390444032224389

http://orcid.org/0000-0003-0723-9260

thatianyspereira@gmail.com

Adeilma Fernandes de Souza

Universidade Federal de Campina Grande, Brasi

http://lattes.cnpq.br/4043789339504825

http://orcid.org/0000-0002-7826-9128

adeilmafsousa@gmail.com

Edja Daise Oliveira Barbosa (1D

Universidade Federal de Campina Grande, Brasil

http://lattes.cnpq.br/5911826557451162

http://orcid.org/0000-0002-8235-363X

edjadayse@hotmail.com

6

DOI: 10.6008/CBPC2318-2881.2019.003.0001
Marcio Frazão Chaves (ii)

Universidade Federal de Campina Grande, Brasi

http://lattes.cnpq.br/5314751391292608

http://orcid.org/0000-0002-0341-2882

marciochavesufcg@gmail.com
Referencing this:

PEREIRA, T. S.; SOUZA, A. F.; BARBOSA, E. D. O.; CHAVES, M. F.. Avifauna alojada nos CETAS/IBAMA nos estados do Rio Grande do Norte e Paraíba, Brasil. Nature and Conservation, v.12, n.3, p.1-10, 2019. DOI: http://doi.org/10.6008/CBPC2318-2881.2019.003.0001 


\section{INTRODUÇÃO}

A utilização da fauna pode ser descrita desde os longínquos tempos, onde vários animais ou parte deles (ossos, dentes, etc.) foram utilizados essencialmente como fonte proteica, e aos poucos estabelecendose em uma dimensão mais ampla, pelo uso de vestimentas, práticas ritualísticas e terapêuticas (ALVES et al., 2010). Desde então, essas percepções histórico-culturais foram repassadas por gerações tornando-se presente nos dias atuais, sobretudo, por meio de algumas populações tradicionais. No Brasil, essa conexão e dependência com a fauna através de atividades baseadas pela caça, pesca e o uso mágico-religioso são relacionadas principalmente aos povos indígenas (PEZZUTTI et al., 2009; ALVES et al., 2012). No entanto, a necessidade de sobrevivência a partir dos recursos biológicos se estenderam a outras populações como os seringueiros, caiçaras, ribeirinhos, entre outros (DIEGUES et al., 2001; ROSAS et al., 2007).

Apesar do seu longo histórico de degradação natural, o Brasil é conhecido mundialmente por sua vasta biodiversidade, compreendendo uma grande variedade de biomas e espécies endêmicas (LEAL et al., 2005). No entanto, com a expansão urbana e as progressivas ações antropogênicas, os danos e as intervenções ambientais culminaram no processo de extinção de inúmeras espécies. E mesmo cientes de tal realidade, as degradações ambientais são contínuas e em alguns casos irreversíveis (PRADO et al., 2006). Esse fato pode ser associado à utilização dos recursos biológicos de forma indiscriminada, através do consumo desmoderado, mas justificado muitas vezes pelo seu potencial econômico (VIDOLIM, 2004).

São inúmeros os fatores que têm contribuído para os processos de extinção de várias espécies faunísticas no Brasil. A falta de estratégias e de políticas conservacionistas impõe restrições no gerenciamento desses recursos. Além disso, o contexto social sobre o uso da fauna passou a ser visto como atividade altamente lucrativa, tornando o tráfico de animais uma prática difundida mundialmente (HERNANDES et al., 2006). O tráfico de animais é apontado como a terceira maior atividade ilícita rentável do planeta, superado apenas pelo comércio de armas e drogas (RENCTAS, 2001) podendo movimentar cerca de 2,5 bilhões por ano (DESTRO et al., 2012). No Brasil, essa prática tornou-se uma das principais fontes do contrabando da fauna (HERNANDEZ, 2002).

Com uma fauna extensa e diversificada, a avifauna brasileira representa cerca de $20 \%$ da diversidade de aves no mundo, compondo uma numerosa lista estimada em 1.919 espécies (CBRO, 2015). São visados por possuírem atrativos, destacando-se os seus cantos e a exuberância de suas cores e plumagens. No entanto, consistem em alvos fáceis de caçadores (BEZERRA, 2012). Além disso, é o grupo animal que mais sofre com o tráfico clandestino, representando cerca de $82 \%$ das espécies capturadas ilegalmente em todo o Brasil (RENCTAS, 2001).

Atualmente as espécies entregues voluntariamente ou apreendidas pelo poder público brasileiro são depositados nos Centros de Triagem de Animais Silvestres (CETAS). Os CETAS são órgãos públicos gerenciados pelo Instituto Brasileiro do Meio Ambiente e dos Recursos Naturais (IBAMA) que têm por finalidade receber, triar, recuperar e destinar os animais da fauna silvestre do Brasil apreendidos ou entregues voluntariamente. No Brasil, existem cerca de 20 Centros de Triagem de Animais Silvestres - CETAS (IBAMA, 2019). 
A análise acerca da entrada de animais silvestres em órgãos como o CETAS é de grande relevância, através dos dados analisados se pode diagnosticar a situação faunística do país conhecendo mais a fundo os processos de captura e comércio ilegal em uma determinada região, bem como, os principais destinos e finalidade para estas práticas e se as espécies traficadas são endêmicas e/ou ameaçadas de extinção, servindo como base para trabalhos voltados para a preservação e conservação.

O presente estudo objetivou caracterizar o comércio ilegal de aves silvestres com base no registro de dados dos Centros de Triagem de Animais Silvestres do IBAMA em duas áreas do Nordeste do Brasil - Rio Grande do Norte e Paraíba. Desta forma, buscou-se o conhecimento da avifauna silvestre recolhida nos Centros de Triagem por meio de informações existentes em sistemas gerenciados pelo IBAMA.

\section{MATERIAS E MÉTODOS}

Este trabalho foi desenvolvido nos Centros de Triagem de Animais Silvestres (CETAS) dos Estados do Rio Grande do Norte e Paraíba), no Nordeste do Brasil. O CETAS da Paraíba, localiza-se em João Pessoa, mais especificamente na Floresta Nacional Restinga de Cabedelo. O CETAS do Rio Grande do Norte, está localizado na cidade de Natal (IBAMA, 2019).

A pesquisa foi previamente autorizada pela superintendência do Instituto Brasileiro do Meio Ambiente e dos Recursos Naturais Renováveis (IBAMA) em ambos os Estados investigados. A análise dos dados ocorreu através de consulta ao banco de dados disponibilizado pelo CETAS-RN e pelo CETAS-PB. Foram realizadas visitas a ambos os CETAS (Centro de Triagem de Animais Silvestres) no período de março de 2014 a janeiro de 2015, registrando-se dados quantitativos e sistematizados dos anos de 2005 a 2014 referentes à entrada de espécimes de aves apreendidas (apreensão decorrente de ações fiscalizadoras) ou entregues voluntariamente pela população local. Para obtenção de maiores informações foram realizadas entrevistas com biólogos dos Centros de Triagem de Animais Silvestres do Estado do Rio Grande do Norte e da Paraíba.

A nomenclatura taxonômica das espécies foi registrada de acordo com o Comitê Brasileiro de Registros Ornitológicos (CBRO, 2014). E a avaliação dos status de conservação das espécies ocorreu por meio de consulta a Lista Nacional das Espécies da Fauna Brasileira Ameaçada de Extinção (ICMBIO, 2014), e à base de dados da International Union for Conservation of Nature (IUCN, 2019). A abundância relativa de cada grupo de animais apreendido foi mensurada de acordo com a fórmula: $\mathrm{Na}^{*}(100 / \mathrm{NA})$ onde: $\mathrm{Na}=$ número de indivíduos da espécie e NA = é o número total de aves recolhidas.

\section{RESULTADOS E DISCUSSÃO}

\section{Centro de triagem de animais silvestres do Rio Grande do Norte e Paraíba}

Durante os anos amostrados, a entrada de animais foi classificada em recolhidas e apreendidas, as recolhidas podem ser classificadas em entregas voluntárias: quando a pessoa se dirige ao IBAMA e entrega "seu" animal, e indeferidos: quando não se tem definido se o animal recebido é oriundo de apreensões. As apreendidas são caracterizadas pela captura de animais provenientes de ações ilegais em diversos locais da 
própria região por meio de operações de apreensão da Policia Florestal e IBAMA.

O CETAS-RN apresentou uma totalidade de 14.546 (91\%) de animais apreendidos, 1.028 (6\%) entregues voluntariamente e 505 (3\%) em recolhimentos indeferidos em toda a região. No CETAS-PB o número de apreensões foram notáveis em todos os anos, evidenciando 32.812 (80\%) apreensões, 1.414 (14\%) entregas voluntárias e 1.801 (6\%) para outras classificações de recebimento como: indeterminada ou ausência de dados (Figura 1. a; b)). As apreensões foram as maiores responsáveis por todos os animais depositados. Os estudos de Souza (2014) em Minas Gerais e Moura et al., (2012) no Piauí, obtiveram resultados semelhantes.

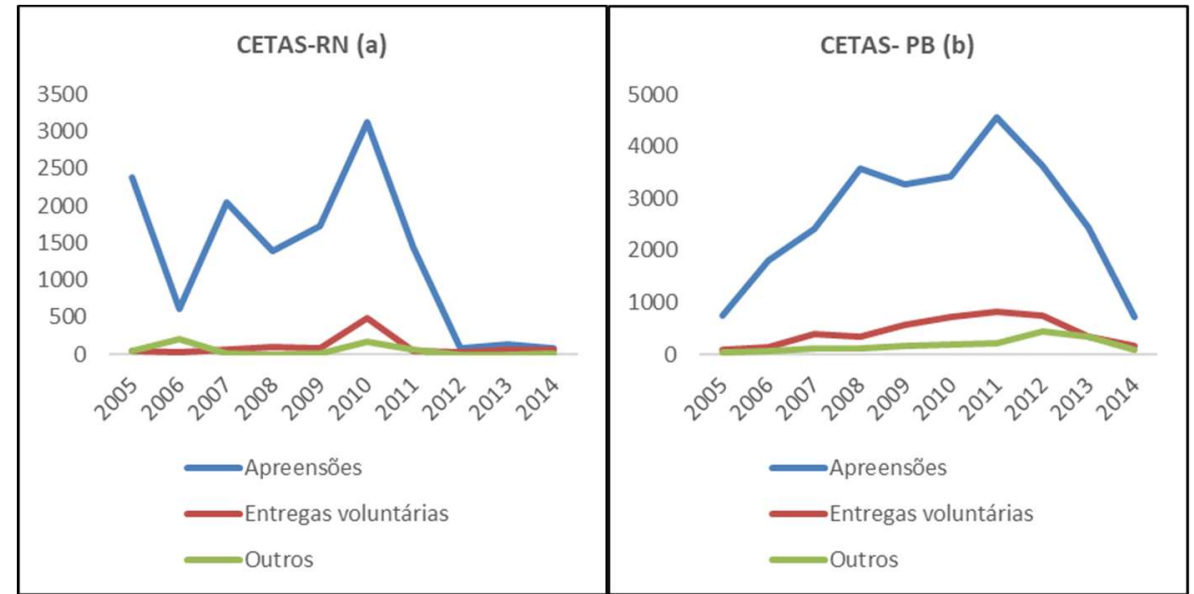

Figura 1: Número de animais recebidos entre os anos de 2005 a 2014 no CETAS-RN (a), CETAS-PB (b)

Para considerar as relações do tráfico de animais silvestres com a entrada de animais no CETAS foi preciso diferenciar a forma de ingresso desses animais. Apesar dos dados sobre a entrada desses animais não descreverem especificamente se houve um aumento ou redução do tráfico durante os anos, esses dados podem estimar através das apreensões realizadas o diagnóstico do tráfico nos Estados do Rio Grande do Norte e Paraíba, fornecendo informações sobre os animais ou espécies mais cobiçadas por esse comércio ilícito (DESTRO et al., 2012).

\section{Centro de triagem de animais silvestres - CETAS Rio Grande do Norte}

A quantidade de aves depositada neste centro foi consideravelmente superior aos demais grupos (Figura 2). A variação em alguns anos representa o número de recebimento desses animais e as operações realizadas pelos órgãos públicos. Ou seja, em 2010 o número de apreensões foi o mais elevado, isso pode ser explicado pelas intensas operações contra o tráfico de animais, aumentando consequentemente o número de apreensões. Em 2012, observou-se que a quantidade de entrada de animais foi menor devido à interdição desse CETAS (ou seja, as atividades antes prestadas aos animais provenientes de fiscalizações e apreensões foram interrompidas pelo IBAMA devido a problemáticas relacionadas a zoonoses em animais silvestres instalados no centro de triagem) influenciando em todo o processo de recebimento de animais

Dentre os animais registrados durante a pesquisa, a abundância de alguns grupos foi mensurada de acordo com sua quantidade em relação aos outros grupos. Répteis e mamíferos demonstraram números relativamente pequenos comparados ao das aves. Foram recebidos um total de 1.363 répteis (9\%), 796 
mamíferos (6\%) e 12.391 aves (85\%).

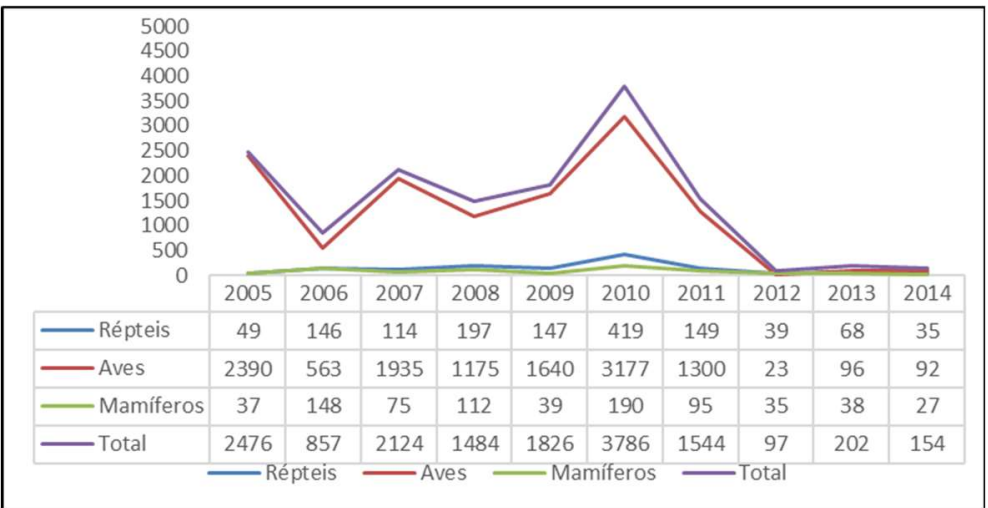

Figura 2: Número de espécimes depositados no CETAS-RN.

Ao longo de quase uma década (2005 a 2014), as aves constituíram o grupo que apresentou os valores mais significativos (CETAS-RN), com aproximadamente $85 \%$ do total de entradas. Esses resultados são semelhantes a outros estudos realizados no semiárido (PAGANO, 2009; DESTRO, 2012). Nos quais, há também uma clara dominância das aves como o grupo mais recebido, representando o equivalente a $82 \%$. Os dados obtidos juntamente com outros trabalhos, sugerem que a avifauna é o grupo que mais sofre com o tráfico ilegal no Brasil (RENCTAS, 2001; MARINI et al., 2005; MOURA et al., 2012). Esse fato pode ser compreendido, em virtude das aves serem alvo constante de caçadores devido a sua importância econômica e sociocultural. Bezerra (2012) argumentou em sua pesquisa a utilização das aves, constatando que a maioria desse grupo geralmente possui um propósito alimentício, econômico ou ornamental.

Dentre as aves apreendidas ao longo dos anos, as ordens mais citadas foram os Passeriformes e Psittaciformes (Figura 3). Essa quantidade foi estimada através da quantidade total de aves em todos os anos. A ordem que se manteve frequente e em maior quantidade com relação às outras, foi Passeriformes apresentando 8.935 (72\%), os Psittaciformes com 1.265 (10\%), os Columbiformes com 1.118 (9\%), Falconiformes 525 (4\%) e as demais ordens com 663 (5\%). Os Passeriformes e Psittaciformes também foram predominantes nos trabalhos de Franco et al. (2002) e Souza (2014), ambos em Minas Gerais. Portanto, a intensa captura dessas aves evidencia a prática crescente da retirada desses animais da natureza para o comércio ilegal. Paixão et al. (2013) relataram essas ordens como as mais encontradas em cativeiros na região paraibana devido a sua variedade de cantos e domesticação.

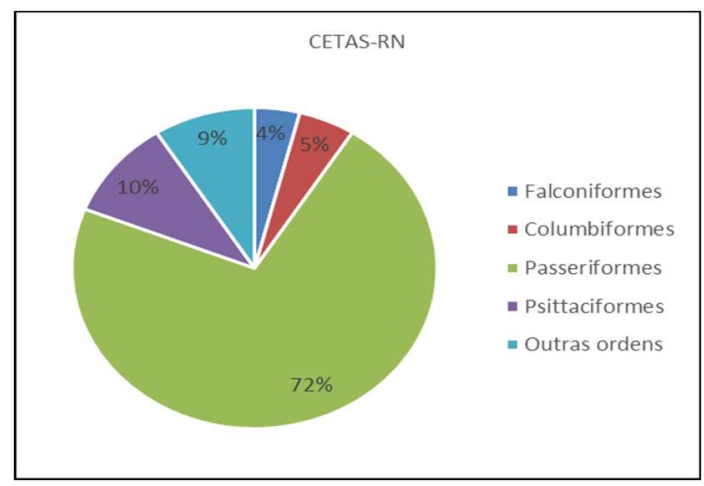

Figura 3: Abundância relativa das ordens das aves recebidas no CETAS-RN. 
Com relação às famílias de aves mais recebidas, as Thraupidae mostraram valores mais consideráveis no CETAS, correspondendo a $48 \%$ de dominância das espécies mais apreendidas (Figura 4). Esses dados também coincidem com as pesquisas de Pagano et al. (2009) na Paraíba, e os Psittacidae também prevaleceram com as espécies mais apreendidas com 15\%. O trabalho de Vilela (2012) em Belo Horizonte também apresenta resultados semelhantes.

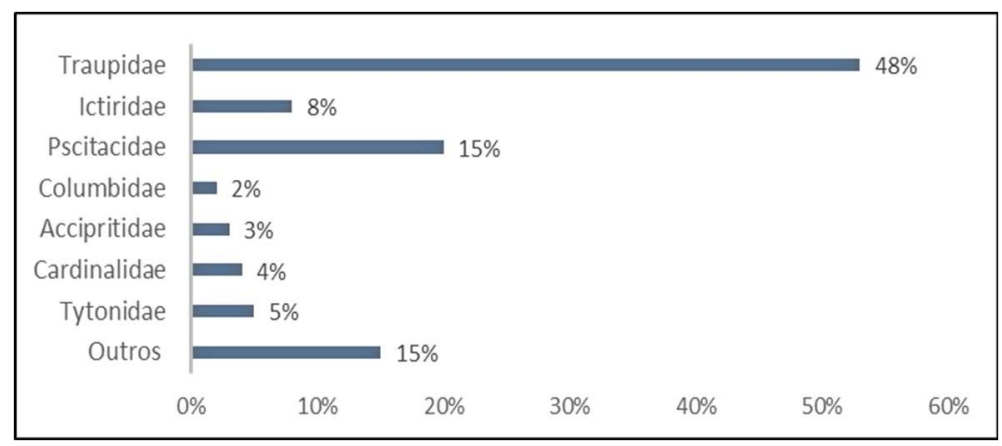

Figura 4: Percentual de famílias de aves recebidas do CETAS-RN.

Os animais apreendidos foram listados pelo critério da frequência durante os anos de 2005 a 2014 (tabela 1). As espécies mais citadas ao longo da pesquisa foram o Sicalis flaveola com 1500 espécies; Paroaria dominicana com 819 espécies e Cyanoloxia brissonii com 740 espécies. Resultados semelhantes foram encontrados em diversos trabalhos (Borges, 2006; Rocha, 2006) reforçando a relação dessas espécies como as mais comercializadas ilegalmente de forma similar a outros estudos.

Tabela 1: Espécies de aves mais apreendidas no CETAS-RN durante os anos de 2005 a 2014. De acordo com a IUCN: N Número de espécimes; IUCN status: PP - Pouco Preocupante; CR - criticamente ameaçado; VU - vulnerável; D/Def. Dados Deficientes e S/Inf. Endêmicas da região Nordeste*.

\begin{tabular}{|l|l|l|l|l|}
\hline Nome do taxón (CBRO 2014) & Nome-popular & IUCN & Total & $\%$ \\
\hline Thraupidae Cabanis, 1847 & & & \\
\hline Sicalis flaveola (Linnaeus, 1766) & Canário-da terra & LC & 1500 & $12 \%$ \\
\hline Paroaria dominicana (Linnaeus, 1758 & Galo de campina* & LC & 1.236 & $10 \%$ \\
\hline Sporophila albogularis (Spix, 1825) & Golinha $*$ & LC & 957 & $8 \%$ \\
\hline Sporophila lineola (Linnaeus, 1758) & Estrelinha & LC & 452 & $4 \%$ \\
\hline Coereba flaveola (Linnaeus, 1758) & Sibite & LC & 391 & $3 \%$ \\
\hline Sporophila bouvreuil (Statius Muller, 1776) & Caboclinho & LC & 249 & $2 \%$ \\
\hline Sporophilas chistacea (Lawrence, 1862) & LC & 158 & $1.2 \%$. \\
\hline Cardinalidae Ridgway, 1901 & Papa capim & & \\
\hline Cyanoloxia brissonii (Lichtenstein, 1823) & & LC & 740 & $6 \%$ \\
\hline Fringillidae Leach, 1820 & Azulão & & \\
\hline Euphonia chlorotica (Linnaeus, 1766) & & LC & 12 & $0.09 \%$ \\
\hline Spinus magellanicus (Vieillot, 1805) & LC & 373 & $3 \%$ \\
\hline Turdidae Rafinesque, 1815 & Guriatã & & \\
\hline Turdus rufiventris Vieillot, 1818 & Pintassilgo & LC & 121 & $1 \%$ \\
\hline Tytonidae Mathews, 1912 & & & & \\
\hline Tyto furcata (Temminck, 1827) & Sabiá laranjeira & LC & 61 & $0.5 \%$ \\
\hline Estrildidae Bonaparte, 1850 & & & & \\
\hline Estrilda astrild (Linnaeus, 1758) & Suidara & LC & 40 \\
\hline Accipitridae Vigors, 1824 & & & $0.3 \%$ \\
\hline Rupornis magnirostris (Gmelin, 1788) & Bico de lacre & 40 \\
\hline Pssittacidae Rafinesque, 1815 & & & $0.3 \%$ \\
\hline Eupsittula cactorum (Kuhl, 1820) & Gavião carijó & LC & 214 \\
\hline Amazona aestiva (Linnaeus, 1758) & & LC & 214 \\
\hline Ara ararauna (Linnaeus, 1758) & Periquito vaqueiro & $2 \%$ \\
\hline
\end{tabular}




\section{Centro de triagem de animais silvestres - CETAS Paraíba}

Na Paraíba, durante os anos avaliados o CETAS recebeu 32.812 animais. A entrada anual desses animais variou durante os anos de 2005 a 2014. O ano de 2005 apresentou números relativamente baixos comparados aos demais, provavelmente o número de operações de fiscalização foi menor, diminuindo a entrada de animais provenientes de apreensões, e a partir de então, houve um aumento gradual na quantidade de espécies recebidas nos anos subsequentes. O ano considerado com o maior índice de recebimento de animais foi o de 2011, demonstrando o aumento de operações de fiscalização pelos órgãos públicos no Estado. Em 2014 a entrada de animais no centro teve um declínio expressivo, isso pode ser justificado pelo fato do IBAMA em conjunto com o CETAS interromperem o recolhimento dos animais oriundos de fiscalizações devido à falta de um médico veterinário.

Os grupos de animais registrados durante a pesquisa foram calculados individualmente através de sua quantidade anual em relação aos demais grupos (Figura 5). Resultando em 2.373 répteis (7\%), 28.570 aves (87\%) e 1.869 mamíferos (6\%). O recebimento de animais provenientes de apreensões apontam o quanto as aves continuam sendo as maiores vítimas do comércio ilegal. Na Paraíba Rocha et al., (2006) relatam que grande parte das aves capturadas são vendidas em feiras livres diretamente (fornecido pelos que capturam) ou indiretamente (através de um intermediário que compra de quem captura).

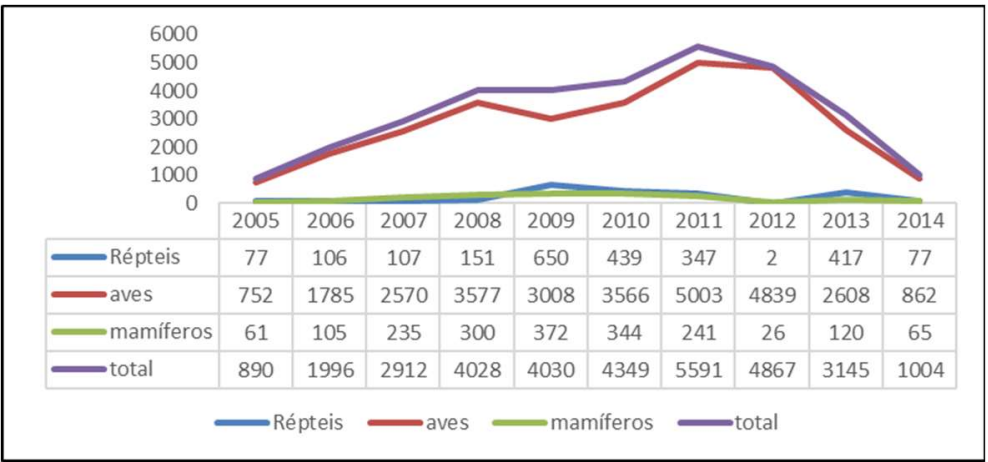

Figura 5: Número de espécimes depositados no CETAS-PB.

Dentre o grupo das aves as ordens que se destacaram como mais recebida no CETAS da Paraíba ao longo de 2005 a 2014 (Figura 6), foram as Passeriformes com 21.634 (75\%), seguida de Psittaciformes com $3.564(12 \%)$, Columbiformes com 994 (3\%), Falconiformes com 437 (1\%) e as demais ordens que se mantiveram inconstantes com 2.698 (9\%). Esses dados se mostraram semelhantes aos já descritos para o CETAS do Rio Grande do Norte. Assemelhando-se aos dados obtidos por Franco et al. (2012) em Minas gerais.

Já as famílias mais apreendidas foram as Thraupidae com 16.912 (59\%) e Psittacidae 3.583 (12\%) (Figura 7). A predominância dessas famílias também foi observado no CETAS-RN. Esses dados são similares aos estudos de Moura e Vilela (2012). As espécies S.flaveola; P.dominicana; C.brissonii; S.albugularis e S.bouvreuil foram as mais apreendidas em ambos com CETAS (tabela 2). Esses resultados também apresentaram similaridade com os dados relatados por, Gogliath et al. (2010) e Pagano et al. (2009). 


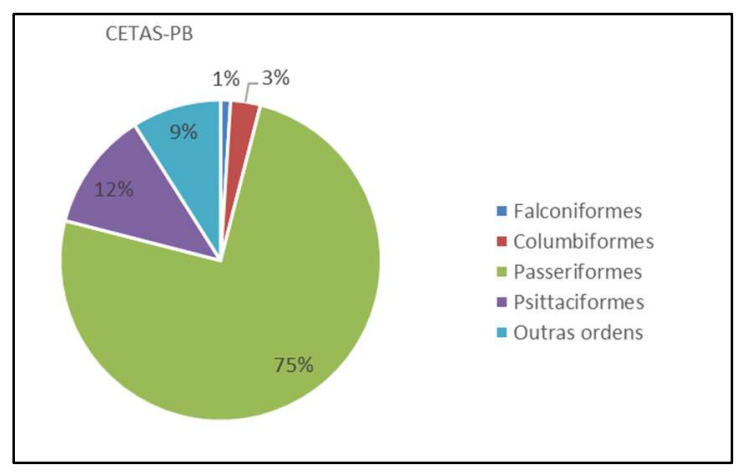

Figura 6: Abundância relativa das ordens das aves que foram recebidas no CETAS-PB.

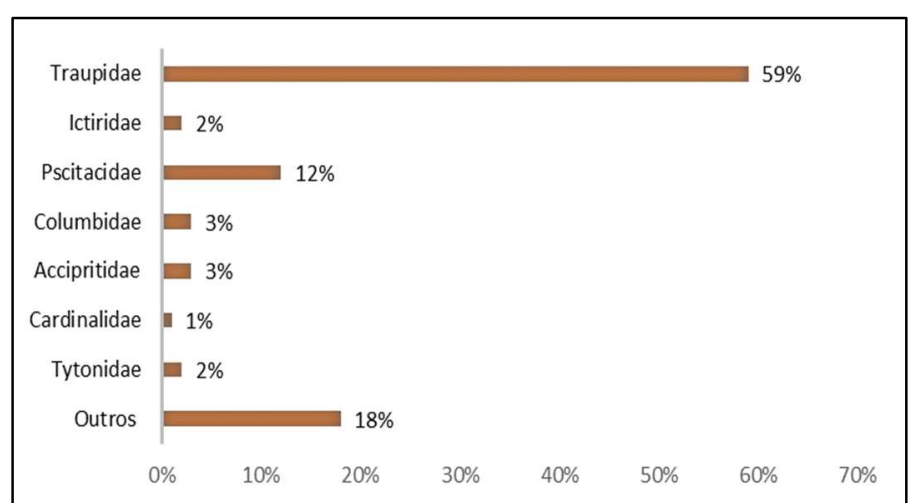

Figura 7: Percentual de famílias de aves recebidas do CETAS RN.

Tabela 1: Espécies de aves mais apreendidas no CETAS-RN durante os anos de 2005 a 2014. De acordo com a IUCN: NNúmero de espécimes; IUCN status: PP - Pouco Preocupante; CR - criticamente ameaçado; VU - vulnerável; D/Def. Dados Deficientes e S/Inf. Endêmicas *

\begin{tabular}{|c|c|c|c|c|}
\hline Nome do taxón (CBRO, 2014) & Nome-popular & IUCN & Total & $\%$ \\
\hline \multicolumn{5}{|l|}{ Thraupidae Cabanis, 1847} \\
\hline Sicalis flaveola (Linnaeus, 1766) & Canário-da-terra & LC & 1.991 & $7 \%$ \\
\hline Paroaria dominicana (Linnaeus, 1758) & Galo de campina * & LC & 1.428 & $5 \%$ \\
\hline Sporophila albogularis (Spix, 1825) & Golado * & LC & 937 & $3 \%$ \\
\hline Sporophila bouvreuil (Statius Muller, 1776) & Caboclinho & LC & 869 & $2 \%$ \\
\hline Sporophila schistacea (Lawrence, 1862) & Papa capim & LC & 551 & $2 \%$ \\
\hline Sporophilal Lineola (Linnaeus, 1758) & Bigode & LC & 452 & $1 \%$ \\
\hline Coereba flaveola (Linnaeus, 1758) & Sibite & LC & 290 & $1 \%$ \\
\hline \multicolumn{5}{|l|}{ Icteridae Vigors, 1825} \\
\hline Chrysomus ruficapillus (Vieillot, 1819) & Corda-negra & LC & 239 & $0.5 \%$ \\
\hline \multicolumn{5}{|l|}{ Turdidae Rafinesque, 1815} \\
\hline Turdus rufiventris Vieillot, 1818 & Sabiá laranjeira & LC & 136 & $0.5 \%$ \\
\hline \multicolumn{5}{|l|}{ Estrildidae Bonaparte, 1850} \\
\hline Estrilda astrild (Linnaeus, 1758) & Bico de lacre & LC & 101 & $0.3 \%$ \\
\hline \multicolumn{5}{|l|}{ Pssittacidae Rafinesque, 1815} \\
\hline Eupsittula cactorum (Kuhl, 1820) & Periquito da Caatinga & LC & 121 & $0.3 \%$ \\
\hline Amazona aestiva (Linnaeus, 1758) & Papagaio verdadeiro & LC & 87 & $0.2 \%$ \\
\hline \multicolumn{5}{|l|}{ Tytonidae Mathews, 1912} \\
\hline Tyto furcata (Temminck, 1827) & Suidara & LC & 82 & $0.2 \%$ \\
\hline \multicolumn{5}{|l|}{ Columbidae Leach, 1820} \\
\hline Columbina squammata (Lesson, 1831) & Rolinha cascavel & LC & 68 & $0.2 \%$ \\
\hline \multicolumn{5}{|l|}{ Fringillidae Leach, 1820} \\
\hline Spinus magellanicus (Vieillot, 1805) & Pintassilgo & LC & 54 & $0.1 \%$ \\
\hline \multicolumn{5}{|l|}{ Accipitridae Vigors, 1824} \\
\hline Rupornis magnirostris (Gmelin, 1788) & Gavião carijó & LC & 52 & $0.1 \%$ \\
\hline \multicolumn{5}{|l|}{ Cardinalidae Ridgway, 1901} \\
\hline Cyanoloxia brissonii (Lichtenstein, 1823) & Azulão & LC & 1207 & $3 \%$ \\
\hline
\end{tabular}

\section{Análise comparativa entre o CETAS do Rio Grande do Norte e Paraíba}

Os dados apresentados nos centros de triagem de animais silvestres nos Estados da Paraíba e do Rio Grande do Norte, foram significativos com relação à contínua entrada de animais. O total de aves recebidas no período amostrado no CETAS-RN registrou 12.391; enquanto que, no CETAS-PB esse valor alcançou 28.750. Pôde-se relacionar esses valores ao número de operações de fiscalização em cada região e ao estado atual de recebimento dos animais em cada centro. As interdições também influenciam diretamente sobre a quantidade de animais apreendidos e recebidos durante o ano de 2014 em ambos os CETAS. 


\section{Diagnóstico do tráfico de animais silvestres}

O aumento do tráfico de animais não pode ser determinado como um fator unitário, atrelando o fortalecimento dessa atividade ilegal apenas a destinação dos animais. A não aplicabilidade da legislação ambiental é um dos pontos que facilitam o crescimento do tráfico de animais, refletindo a necessidade do cumprimento de multas e penas. Campus-Neto (2012) aponta a necessidade da eficiência das punições como uma alternativa, mas, propõe uma menor flexibilização de leis que culminam muitas vezes em prescrições por parte dos infratores.

A melhoria significativa da estrutura dos órgãos públicos responsáveis pela fiscalização, manutenção e recuperação dos animais silvestres é o ponto fulcral para o combate ao tráfico. A falta de investimentos e profissionais capacitados resulta muitas vezes no aumento da comercialização ilegal. Destro (2012) ressalta a necessidade de investimentos nesses órgãos públicos como medidas específicas de controle ao tráfico. A destinação dos animais nos centros de acolhimento desses animais, demonstrou uma situação delicada quanto a permanência e soltura de algumas espécies, é notória a importância de manter condições necessárias para atender, tratar e recuperar os animais para uma possível soltura. Efe et al. (2006), relatou a superlotação desses espaços como uma dificuldade enfrentada em todo o país, visto que existe uma quantidade de CETAS no Brasil insuficiente à demanda de animais apreendidos. Estabelecendo-se como uma problemática nacional persistente, a comercialização ilegal de animais silvestres ainda é tratada como cultural e vista por muitas pessoas com naturalidade. A caça dos animais silvestres com a finalidade comercial fornece uma ampla rede de comércio distribuída por todo o mundo, principalmente entre as regiões brasileiras (RENCTAS, 2001).

\section{CONCLUSÕES}

A pesquisa sobre a entrada de animais nos Centros de Triagem de Animais Silvestres do Rio Grande do Norte e Paraíba de 2005 a 2014 demonstrou que a relação do tráfico com os dados referentes apenas à entrada de animais nesses centros é uma análise pouco aproximada da realidade, visto que, esse diagnóstico só pode ser caracterizado através do número de operações constantes de fiscalização.

Através dos valores históricos obtidos das apreensões ao longo de uma década, pôde-se estimar a situação do tráfico da avifauna nos estados da Paraíba e do Rio Grande do Norte. A falta de investimentos nos centros de triagem de animais silvestres somada à carência de destinação dos animais apreendidos e profissionais capacitados contribuiu significativamente com o aumento do tráfico ilegal de animais silvestres.

No CETAS de ambos os estados, as apreensões foram a forma mais comum de entrada dos animais, sendo as aves o grupo predominante em todos os anos avaliados. Dentre as aves, as ordens mais recebidas e apreendidas foram os Passeriformes e os Psittaciformes. As famílias constantes no tráfico de animais silvestres foram Thraupideae e Psittacidae. As espécies mais apreendidas foram o S. flaveola (canário-daterra), P. dominicana (galo-de- campina) e C. brissonii (azulão). Já as espécies endêmicas da caatinga mais frequentes durante os anos foram $P$. dominicana (galo-de-campina), E. cactorium (periquito-da-caatinga) e 
S. albogularis (golinha).

\section{REFERÊNCIAS}

ALVES, R. R. N.; GONÇALVES, M. B. R.; VIEIRA, W. L. S.. Caça, uso e conservação de vertebrados no semiárido Brasileiro. Revista Tropical Conservation Science, v.5, n.3, p.394-416, 2012. DOI: https://doi.org/10.1177/194008291200500312

ALVES, R. R. N.; SOUTO, W. M. S.; MOURÃO, J. S.. A Etnozoologia no Brasil. Recife: NUPEEA, 2010.

BEZERA, D. M. M.; ARAUJO, H. F. P.; ALVES, R. R. N.. Captura de aves silvestres no semiárido brasileiro: técnicas cinegéticas e implicações para conservação. Revista Tropical Conservation Science, v.5, n.1, p.50-66, 2012. DOI: https://doi.org/10.1177/194008291200500106

BORGES, R. C.; OLIVEIRA, A.; BERNARDO, N.; COSTA, R. M. $M$. C.. Diagnóstico da fauna silvestre apreendida e recolhida pela Polícia Militar de Meio Ambiente de Juiz de Fora, MG (1998 e 1999). Revista Brasileira de Zoociências, v.8, n.1 p.23-33, 2006. DOI: https://doi.org/10.34019/2596$\underline{3325.2006 . v 8.24152}$

BRASIL. Ministério do Meio Ambiente. Portaria n. 444, de 17 de dezembro de 2014. Táxons ameaçados no brasil (espécies terrestres e mamíferos aquáticos). Brasília: DOU, 2014.

CAMPOS NETO, A. A. M.. O tráfico de animais. Revista da Faculdade de Direito, São Paulo, v.106, n.1, p.307-347, 2012. DOI: http://doi.org.10.11606/issn.23188235.v106i106-107p307-347

CBRO. Comitê Brasileiro de Registros Ornitológicos. Listas das aves do Brasil. 11 ed. CBRO, 2014.

DESTRO, G. F. G.. Esforços para o combate ao tráfico de animais silvestres no Brasil. Biodiversity, 2012.

DIEGUES, A. C. S.; ARRUDA, R. S. V.. Saberes tradicionais e biodiversidade no Brasil. Brasília: Ministério do Meio Ambiente, 2001.

FRANCO, M. R.; CÂMARA, F. M.; ROCHA, D. C. C.; SOUZA, R. M.; OLIVEIRA, N. J. F.. Animais silvestre apreendidos no período de 2002 a 2007 na macrorregião de Montes Claros, Minas Gerais. Revista Enciclopédia Biosfera, v.8, n.14, p.1007-1018, 2012.

GOGLIATH, M.; BISAGGIO, E. L.; RIBEIRO, L. B.; RESGALLA, A. E.; BORGE, R. C.. Avifauna apreendida e entregue voluntariamente ao Centro de Triagem de Animais Silvestres (CETAS) do Ibama de Juiz de Fora, Minas Gerais. Atualidades Ornitológicas, 2010.

HERNANDEZ , E. F. T.. Das redes e do tráfico de animais. Geografia, Londrina, v.11, n.2, p.271, 2002. DOI: http://dx.doi.org/10.5433/2447-1747.2002v11n2p271

HERNANDEZ, E. F.; CARVALHO, M. S.. O tráfico de animais silvestres no Estado do Paraná. Acta Scientiarum. Human and Social Sciences, v.28, n.2, p.257-266. DOI: http://dx.doi.org/10.4025/actascihumansoc.v28i2.16

IBAMA. Instituto Brasileiro do Meio Ambiente e dos Recursos Naturais Renováveis. Centros de Triagem de
Animais Silvestres. Unidades dos Centros de Triagem de Animais Silvestres (CETAS). Brasília: IBAMA, 2019.

IUCN. International Union for Conservation of Nature. The IUCN Red List of Threatened Species. Version 2019-2. Gland: IUCN, 2019.

LEAL, I. R.; TABARELLI, M.; SILVA, J. M. C.. Ecologia e Conservação da Caatinga. 2 ed. Recife: Universitária da UFPE, 2005.

MARINI, M. A.; GARCIA, F. I.. Conservação de aves no Brasil. Megadiversidade, v.1, n.1, p.95-101, 2005.

MOURA, S. G.; PESSOA, F. B.; OLIVEIRA, F. F.; LUSTOSA, A. H. M.; SOARES, C. B.. Animais Silvestres Recebidos pelo Centro de Triagem do Ibama no Piauí no Ano de 2011. Enciclopédia Biosfera, Goiânia, v.8, n.15, p.1748-1761, 2012.

PAGANO, I. S. A.; SOUSA, A. E. B. A.; WAGNER, P. G. C.; RAMOS, R. T. C.. Aves depositadas no centro de triagem de animais silvestres do IBAMA na Paraíba: uma amostra do tráfico de aves silvestres no estado. Ornithologia, v.3, p.132144, 2009.

PAIXÃO, R. M. C.; SILVESTRE, L. C.; PESSOA, T. S. A.; SOUSA, A. E. B. A.. Entre saberes e observações: a manutenção em cativeiro de Passeriformes silvestres em uma comunidade da Zona da Mata Paraibana. Atualidades Ornitológicas On-line, n.174, p.54-59, 2013.

PEZZUTI, J.; CHAVES, R. P.. Etnografia e manejo de recursos naturais pelos índios Deni, Amazonas, Brasil. Revista Acta Amazonica, Manaus, v.39, n.1, p.121-138, 2009. DOI: http://dx.doi.org/10.1590/S0044-59672009000100013

PRADO, R.; FERREIRA, T. A.; GUIMARÃES, A. M. S.; FARIA, Z.. Efeito da implantação de rodovias no cerrado brasileiro sobre a fauna de vertebrados. Acta Scientiarum: Biological Sciences, v.28, n.3, p.237-241, 2006. DOI: http://doi.org.10.4025/actascibiolsci.v28i3.215

RENCTAS. $1^{\circ}$ Relatório nacional sobre o tráfico de fauna silvestre. Rede Nacional de Combate ao Tráfico de Animais Silvestres. Brasília: RENCTAS, 2001.

ROCHA, M. S. P.; CAVALCANTI, P. C. M.; SOUSA, R. L.; ALVES, R. R. N.. Aspectos da comercialização ilegal de aves nas feiras livres de Campina Grande, Paraíba, Brasil. Revista de Biologia e Ciências da Terra, v.6, n.2, p.204-21, 2006.

ROSAS, G. K. C.; DRUMOND, P. M.. Caracterização da Caça de Subsistência em Dois Seringais Localizados no Estado do Acre (Amazônia, Brasil). Rio Branco: Embrapa, 2007.

SOUSA, T. O.; VILELA, D. A. R.; CÂMARA, B. G. O.. Pressões Sobre A Avifauna Brasileira: Aves Recebidas Pelo Cetas/Ibama, Belo Horizonte, Minas Gerais. Ornithologia, v.7, n.1, p.1-11, 2014.

VIDOLIN, G. P.; MANGINI, P. R.; BRITTO, M. M.; MUCHAIL, M. C.. Programa Estadual de Manejo de Fauna Silvestre Apreendida - Estado do Paraná, Brasil. Revista Cadernos da biodiversidade, v.4, p.37-49, 2004.

A CBPC - Companhia Brasileira de Produção Científica (CNPJ: 11.221.422/0001-03) detém os direitos materiais desta publicação. Os direitos referem-se à publicação do trabalho em qualquer parte do mundo, incluindo os direitos às renovações, expansões e disseminações da contribuição, bem como outros direitos subsidiários. Todos os trabalhos publicados eletronicamente poderão posteriormente ser publicados em coletâneas impressas sob coordenação da Sustenere Publishing, da Companhia Brasileira de Produção Científica e seus parceiros autorizados. Os (as) autores (as) posteriormente ser publicados em coletâneas impressas sob coordenação da Sustenere Publishing, da Companhia Brasileira de Produção Científica e seus para
preservam os direitos autorais, mas não têm permissão para a publicação da contribuição em outro meio, impresso ou digital, em português ou em tradução. 